\title{
METALLIC NANOPARTICLES GENERATION BY REPETITIVE PULSED LASER FOR APPLICATIONS IN BIO-MEDICINE
}

\author{
N. Restuccia ${ }^{a, *}$, L. Silipigni ${ }^{a}$, M. Cordaro ${ }^{b}$, AND L. Torrisi ${ }^{a}$ \\ a Dipartimento di Scienze Matematiche e Informatiche, Scienze Fisiche e Scienze della Terra, MIFT, Università \\ di Messina, V.le F. Stagno d'Alcontres 31, 98166 S. Agata, Messina, Italy \\ ${ }^{b}$ Dip.to di Scienze Chimiche, Biologiche, Farmaceutiche e Ambientali, Università di Messina, Italy \\ * nrestuccia@unime.it
}

\begin{abstract}
An Nd:YAG pulsed laser operating at the $1064 \mathrm{~nm}$ wavelength, the $3 \mathrm{~ns}$ pulse duration, the $10^{10} \mathrm{~W} / \mathrm{cm}^{2}$ intensity and the $10 \mathrm{~Hz}$ repetition rate is employed to irradiate biocompatible metallic targets based on $\mathrm{Au}, \mathrm{Bi}$ and $\mathrm{Ag}$ placed in water. The laser-matter interaction produces nanometric spherical particles. The concentration of the solution with nanoparticles is controllable by the laser parameters, the ablative emission process, the irradiation time and the water's volume. Generally, nanoparticles of about $10 \mathrm{~nm}$ in size and concentrations of the order $(0.1 \div 10) \mathrm{mg} / \mathrm{ml}$ are prepared to be injected in cell cultures or in living systems (mice). The nanoparticles introduction in the extra and intracellular cellular liquids improves the bio-imaging of the tissue and organs by using fluorescence techniques. Moreover, if these nanoparticles are concentrated in tumour cells, they make possible high efficiency radio-therapy and thermal-therapy treatments, as it will be presented and discussed.
\end{abstract}

Keywords: Laser ablation in water; nanoparticles; imaging; therapy.

\section{Introduction}

Recently nanoparticles (NPs) are more and more used for many applications in different scientific fields, such as physics, chemistry, materials science and biomedicine. Advancements have been obtained in the applications of heavy metallic elements nanoparticles in the field of biological and medical applications with the aim to enhance both the diagnostic imaging contrast, by using these nanoparticles such as contrast medium, and the targeting of diseased tissues owing to the increase in the effective atomic number before they are submitted to radiotherapy [1]. By using nanoparticles as markers to control the metabolic phases of internalization in the cells, nanotechnology applied to life sciences has led to a better understanding of the cellular and molecular processes and factors that cause a given disease. By means of different techniques, such as the electronic microscopy, the optical absorption spectroscopy and the X-ray spectroscopy, it is possible to follow the uptake and the decay of nanoparticles often functionalized with specific bio-molecules.

Among all the diagnostic imaging modes, the Xray imaging techniques are the most used for their simplicity, high spatial resolution and low cost. The improvement of these techniques strongly depends on the development of new X-ray contrast agents. Up to now, the clinically used contrast medium consists of small iodine molecules in liquid solutions that allow highlighting the functionality of particular organs. However, the iodine medium can cause serious side effects due to excessive repeated doses necessary to achieve a good image contrast. Recently, metallic nanoparticles (NPs) have been used as contrast agents for the radiological imaging [2]. To this reason there is a growing interest in the development of alternative contrast agents with a high atomic number and a high X-ray absorption, such as for example silver, gold and bismuth nanoparticle solutions.

A special interest is devoted to the use of functionalized nanoparticles able to cross the cell membranes and to diffuse in the intra-cellular liquid up to the nucleus [3]. The aim is that to introduce high atomic number (Z) nanoparticles in the cancer cells to enhance the electronic density of the medium and the possibility to release higher quantities of energy coming from radiations used for radiotherapy (X-rays, electron and ion beams) [4]. The main purpose of radiotherapy is to maximize the radiation dose delivered to the tumors and at the same time minimize the level of damage to surrounding healthy tissues by reducing the dose they receive.

A complementary approach to potentially achieve this goal involves the localization of high atomic number $(\mathrm{Z})$ elements and compounds in the target tumor tissue before irradiation. The presence of high $\mathrm{Z}$ atoms in the target leads to an increase in the cross-section of the photoelectric effect (it strongly depends on the $\mathrm{Z}$ value) and to the generation of low-energy free radicals (photoelectrons and Auger electrons). These lowenergy free radicals have high rates of linear energy transfer (LET) and, consequently, can cause DNA damage through the generation of reactive oxygen species. NPs have several advantages over iodine because they have a higher Z-number and therefore a higher probability of photoelectric interactions.

Silver, gold and bismuth are biocompatible metals that can be obtained as nanometric particles in bio- 
logical solutions. They have high electron density, low toxicity and they easily tend to chemically bind to specific bio-molecules in order to be vehicled trough the blood flux. Their production by laser ablation in water is consolidated by years and it is due to the ablation of atoms from the solid surface, to their energetic interaction in water, inside the plasma generated at the solid-liquid interface, and to their nucleation in nanoparticles with a dimension depending on the laser parameters, irradiation conditions and nature of the used liquid. The mass absorption coefficients of $\mathrm{X}$-ray and the stopping power for electrons and ions versus the energy show a significant increase on the values for such metallic elements with respect to the light biological tissues composition, constituting high contrast medium, when introduced in biological environment, and target elements in cancerous biological tissues for appropriate radiotherapy expositions.

\section{Materials and Methods}

Metallic nanoparticles of Ag, Au and Bi were prepared by using the technique of the pulsed laser ablation in liquids. A Nd:YAG laser was employed at the 1064 $\mathrm{nm}$ wavelength, the $3 \mathrm{~ns}$ pulse duration, the $100 \mathrm{~mJ}$ maximum pulse energy, the $1 \mathrm{~mm}^{2}$ focused spot and the $10 \mathrm{~Hz}$ repetition rate. The metal, in the form of sheets of about $2 \mathrm{~cm}^{2}$ surface and $1 \mathrm{~mm}$ thickness, was placed on the bottom of a glass beaker. A volume of $(5 \div 10) \mathrm{ml}$ of distilled water was employed to generate the solution. Generally the metal surface was placed $4 \mathrm{~mm}$ under the free level of the water in the container. The laser light was perpendicular to the free water surface and it was focused on the metallic target surface. In order to avoid generation of deep craters during the repetitive laser shots, the beaker glass was placed on a rotating and sliding platform to be able to hit the fresh surface.

The ablation yield, in terms of ablated mass per laser pulse, increased about linearly with the laser fluence $\left(\mathrm{J} / \mathrm{cm}^{2}\right)$ and has been controlled by the laser fluence and the solution concentration, because the increment of the laser fluence enhances the ablation yield while an higher concentrated solution absorbs laser energy and decreases the ablation yield [2]. Generally, for the repetition rate, we used exposition times of the order of $(10 \div 30)$ min, i.e. a number of laser shots within $6 \times 10^{3}$ and $1.8 \times 10^{4}$. A scheme of the used experimental set-up is reported in Figure 1a. Figure $1 \mathrm{~b}$ shows a photo of the experimental apparatus.

The metallic nanoparticles can be produced by different laser wavelengths, however, the IR radiation was chosen to have higher ablation yield with respect to visible and UV radiations, in agreement with the literature [5].

By using a $70 \mathrm{~mJ}$ pulse energy and an irradiation time of $10 \mathrm{~min}$ and covering the metallic sheet surface with $4 \mathrm{~mm}$ liquid, $10 \mathrm{ml}$ in volume, the ablated mass was of $1.2 \mathrm{mg}, 1.0 \mathrm{mg}$ and $3.8 \mathrm{mg}$, for $\mathrm{Ag}, \mathrm{Au}$ and $\mathrm{Bi}$, respectively. The IR laser ablation approximately is inversely proportional to the boiling point of the elements, which is $961^{\circ} \mathrm{C}, 1063^{\circ} \mathrm{C}$ and $271^{\circ} \mathrm{C}$ for $\mathrm{Ag}$, $\mathrm{Au}$ and $\mathrm{Bi}$, respectively. In such conditions the produced solution will have a concentration of $1.2 \mathrm{mg} / 10$ $\mathrm{ml}, 1 \mathrm{mg} / 10 \mathrm{ml}$ and $3.8 \mathrm{mg} / 10 \mathrm{ml}$ for $\mathrm{Ag}$, $\mathrm{Au}$ and $\mathrm{Bi}$, respectively.

The production of nanoparticles in a liquid at high concentration promotes their interaction coalescence producing agglomerated and micrometric particles that may precipitate in the solution.
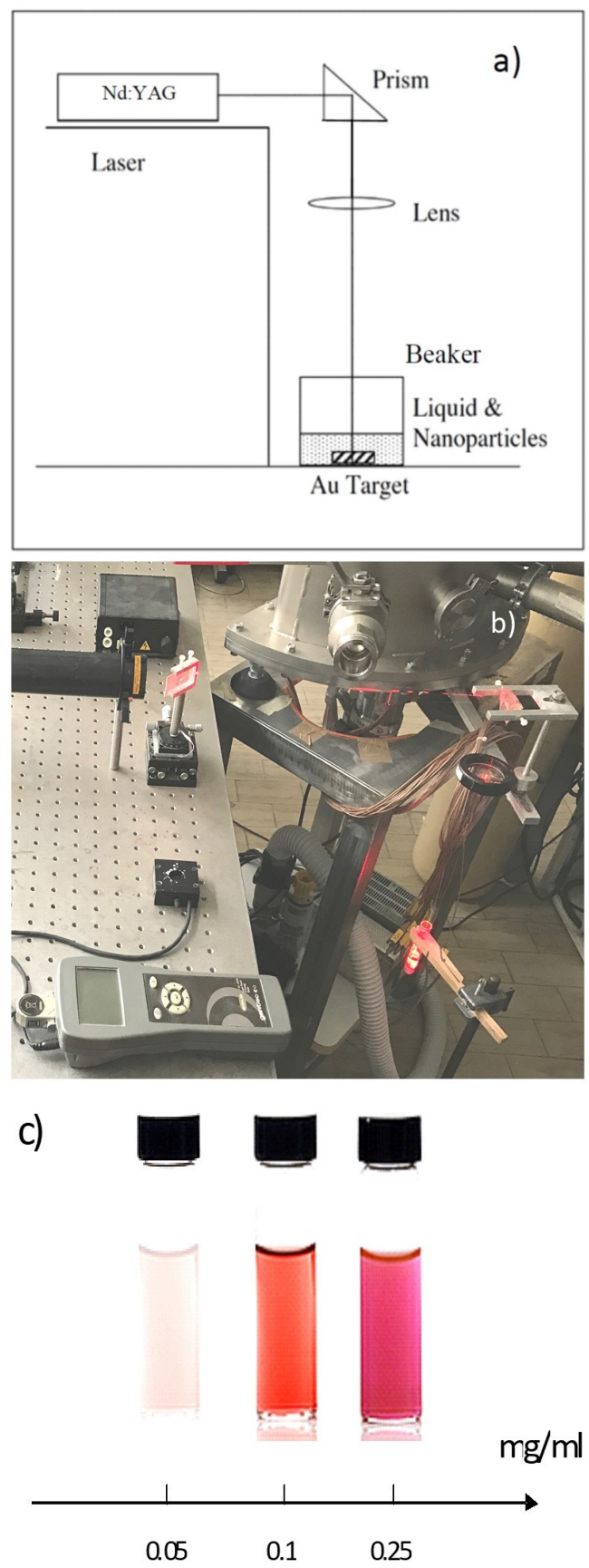

Figure 1. Laser Ablation in liquid: (a) scheme of the experimental set up, (b) its photo and (c) colour of the solution containing Au NPs in different concentrations. 

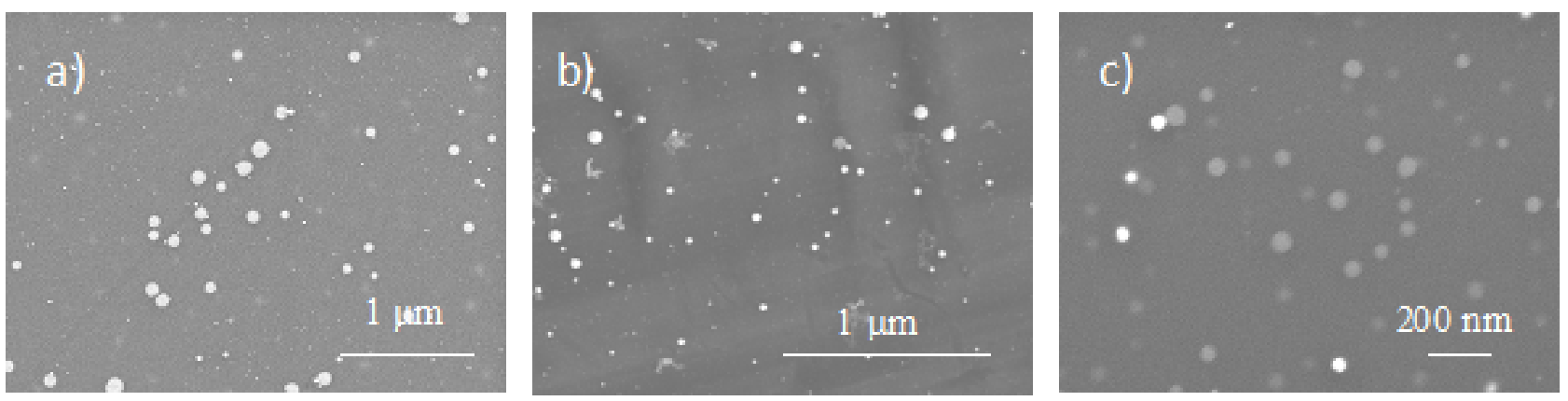

Figure 2. SEM images of nanoparticles of $\mathrm{Ag}(\mathrm{a}), \mathrm{Au}(\mathrm{b})$ and $\mathrm{Bi}(\mathrm{c})$ prepared by laser ablation in water.

Such effect increases with the particles concentration, time and temperature. In order to reduce the nanoparticles coalescence effect a biocompatible surfactant (cap agent) was added, in the form of sodium citrate $\left(\mathrm{Na}_{3} \mathrm{C}_{6} \mathrm{H}_{5} \mathrm{O}_{7}\right)$, by using a $1 \mu \mathrm{g} / 10 \mathrm{ml}$ of surfactant concentration in water. In this way we have stabilized the nanoparticles and avoided a fast their coalescence. The color of the solution changes both with the particles nature and their concentration: it is grey for $\mathrm{Ag}$ and $\mathrm{Bi}$ solutions and red for gold and these colors become more and more intense as the concentration of the solution increases. In particular, Figure 1c shows the colour changes with the Au NPs concentration in distilled water.

Different physical techniques were employed to characterize the produced nanoparticles such as scanning electron (SEM) microscopy, energy dispersive X-ray (EDX) characteristic fluorescence by using electron ionization, X-ray diffraction (XRD) to obtain crystallographic information, and absorbance spectroscopy in the UV- Vis-NIR regions.

The measurements of optical absorbance due to surface plasmon resonance (SPR) analysis were obtained using transmitted light through the solution versus the wavelength and it is affected by scattering effects due to the thin layer crossed in the $4 \mathrm{~mm}$ inner cuvette size.

Measurements of uptake and decay of the used solutions containing nanoparticles were performed in living systems (mice) by using the $\mathrm{X}$-ray fluorescence of the element induced by a photon ionization of a 45 $\mathrm{kV}$ X-ray tube (Bruker-in vivo MS-FX PRO system).

\section{Results}

The SEM images of the metallic nanoparticles have indicated their spherical shape and a dimension ranging between about $5 \mathrm{~nm}$ and $50 \mathrm{~nm}$ in the three cases. The dimensions distribution, the shape and the study of little nanoparticles aggregates was performed by using a $20 \mathrm{kV}$ SEM to observe nanoparticles deposited on a silicon surface. Typical SEM images are reported in Figure 2 for silver (a), gold (b) and bismuth (c). The prolonged laser irradiation up to about $30 \mathrm{~min}$ determines two size distributions of nanoparticles in the liquid, one at about 20-30 nm diameter and another at about 5-10 nm diameter. This is due to the laser absorption in the high concentrated solution that produces further ablation and fragmentation of nanoparticles in smaller dimensions.

The presence of the metallic nanoparticles in the solution induces surface plasmon resonance (SPR) absorption effects when it is illuminated in the VisNIR wavelength regions. In fact, the solution shows absorption bands at wavelengths depending strongly on the particles shape, dimensions and concentration and on the nature of liquid medium. The resonant absorption is due to the dipole induction by light and to electron oscillations on the surface of metallic nanoparticles absorbing energy from the incident light.
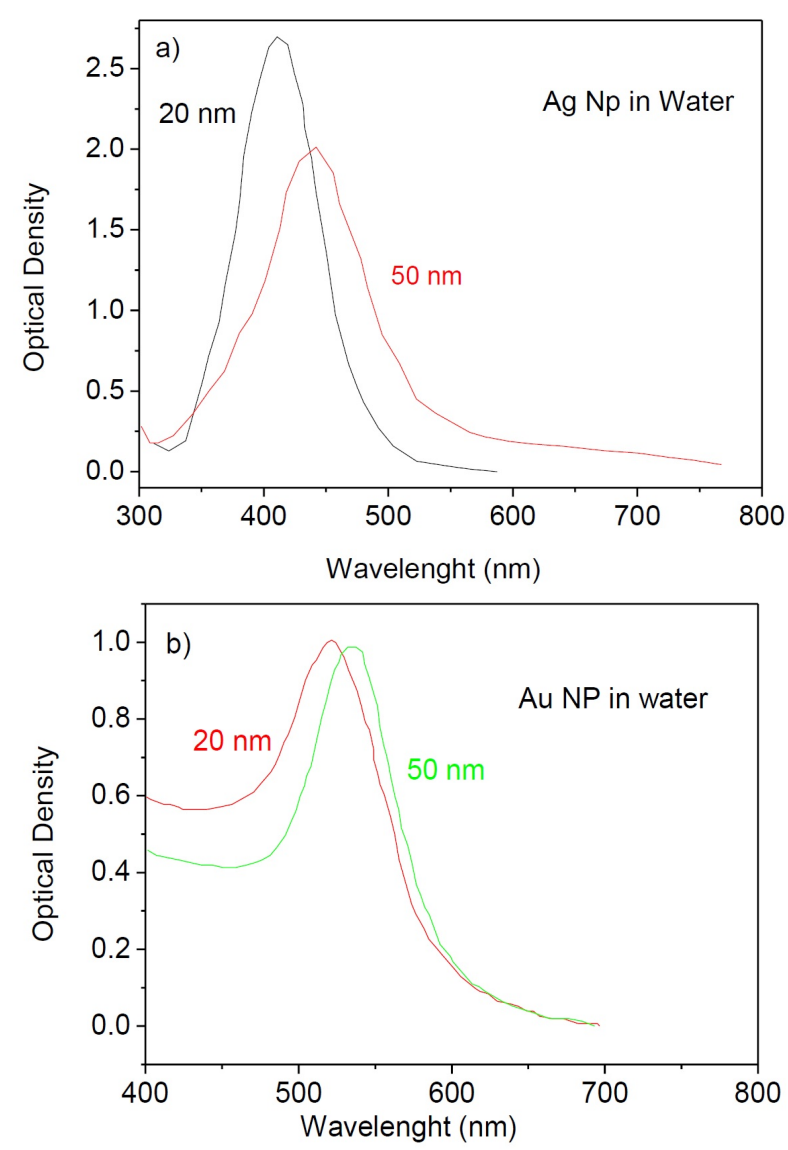

Figure 3. Typical optical absorbance spectra for Ag (a) and $A u(b) N P s$, as a function of wavelength and the spherical nanoparticles diameter. 
Figure 3 reports two plots relative to the typical absorption bands for $\mathrm{Ag}$ (a) and $\mathrm{Au}$ (b) NPs versus the wavelength. It is possible to observe that for spherical particles with $20 \mathrm{~nm}$ diameter the absorption SPR resonance occurs at about $400 \mathrm{~nm}$ for silver and 520 $\mathrm{nm}$ for gold, and that increasing the NPs dimension the absorption peak shifts towards higher wavelengths. For bismuth the peak is less intense than for Ag and $\mathrm{Au}$ and occurs at about $270 \mathrm{~nm}$, according to the literature [6].

The peak shape is typical of $\mathrm{Ag}, \mathrm{Au}$ and $\mathrm{Bi}$ NPs in distilled water showing the SPR band peak and laterally the absorption values vs. wavelength due to other mechanisms. It depends strongly on the nanoparticle nature, size and concentration and on the type of liquid, as reported in the literature [7].

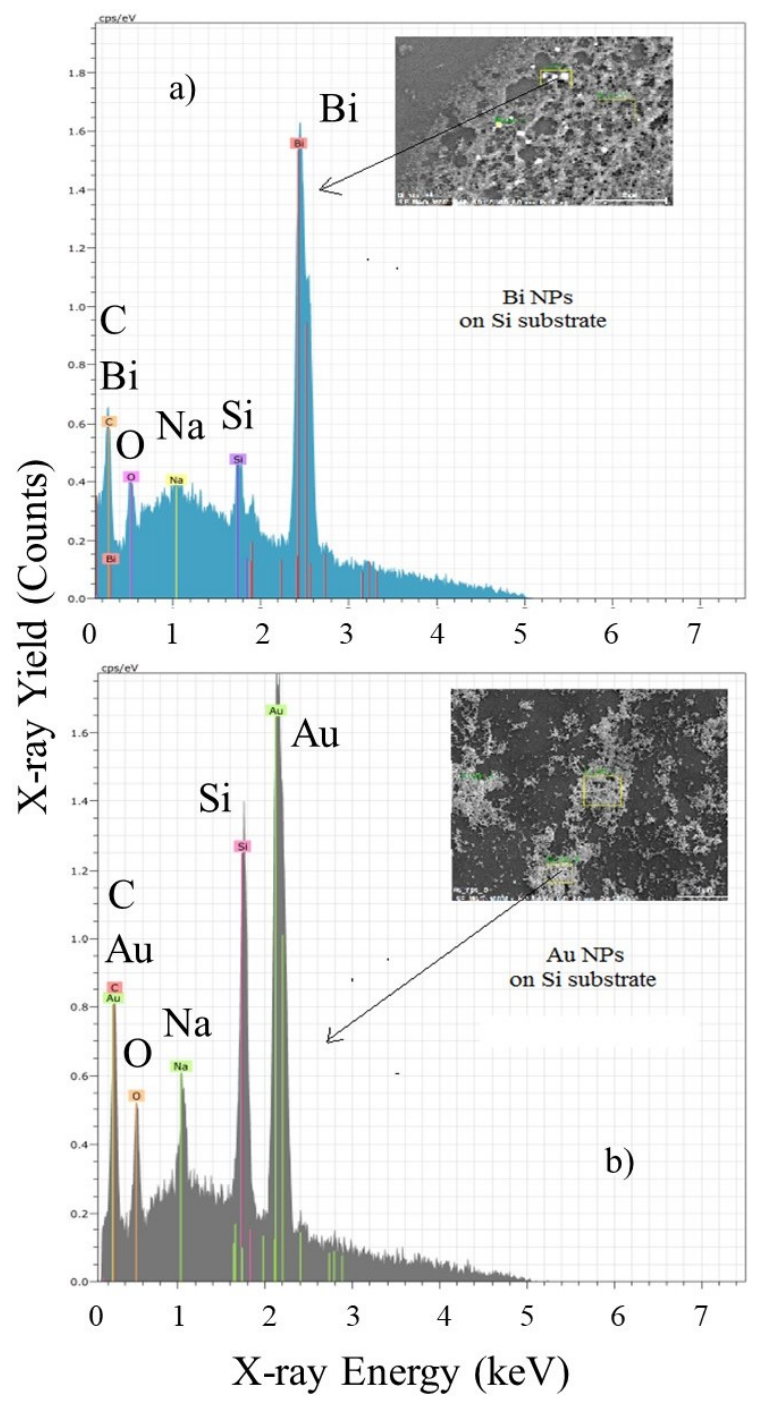

Figure 4. EDX spectra for Bi (a) and Au (b) NPs deposited on a Si substrate: the insets show the analyzed portion.

Figure 4 illustrates EDX spectra, carried out during SEM analysis with a $5 \mathrm{keV}$ electron beam and relative to a group of Bi-NPs (a) and Au-NPs (b) reported in the SEM image of the inset. The Bi NPs EDX spectrum (Figure 4a) displays the characteristic Mlines of $\mathrm{Bi}$, at about $2.4 \mathrm{keV}(\mathrm{M} \alpha)$, the $\mathrm{K}$-line of Si due to the silicon substrate, the $\mathrm{O}$ and $\mathrm{C}$ K-lines derived from contamination of the substrate. The signal from Na comes from the surfactant compounds used at a very low concentration to stabilize the Bi NPs. The $\mathrm{O}$ low presence indicates that the $\mathrm{Bi}$ is metallic and it is very little oxidized, as confirmed by our Raman spectroscopy, XRD, and XPS spectroscopy analyses and in agreement with the literature $[8,9]$.
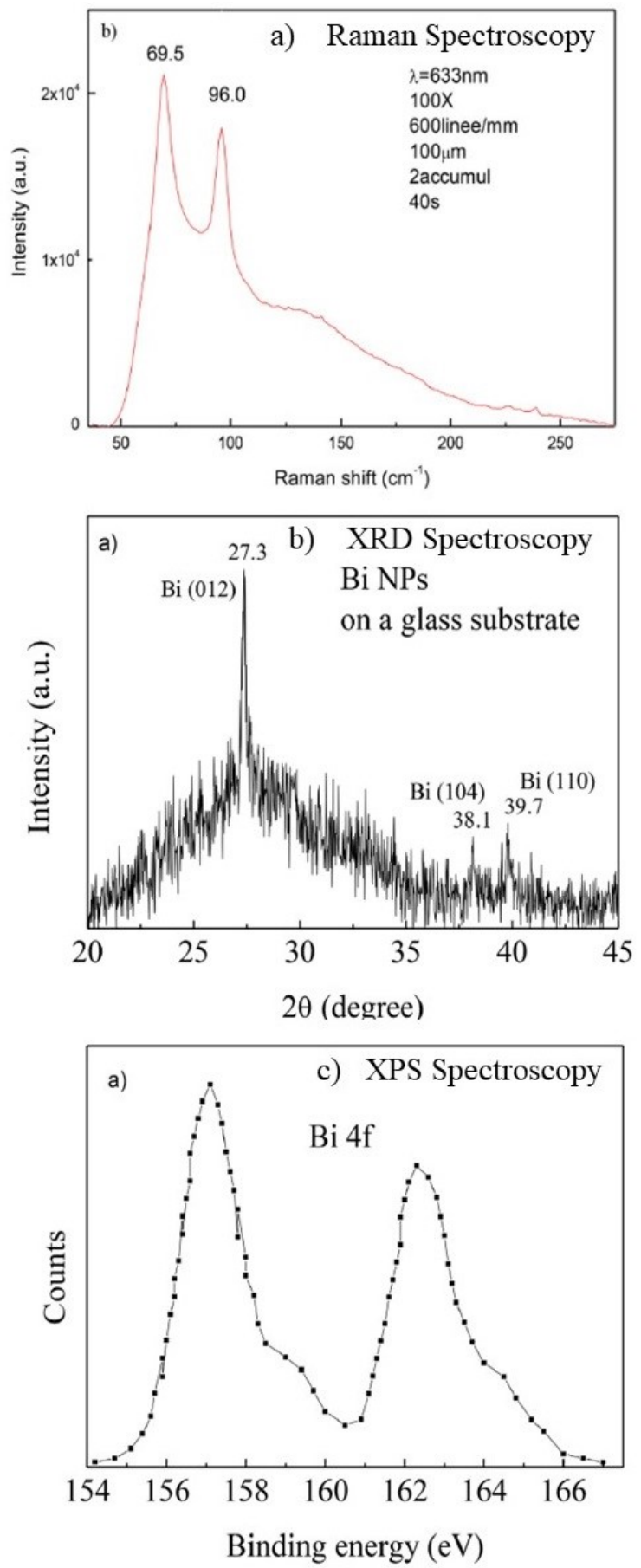

Figure 5. Raman spectroscopy (a), X-ray diffraction analysis (b) and X-ray photoelectron spectroscopy (c) of the prepared Bi-NPs films. 
In particular, the characteristic first order Raman modes of rhombohedral bismuth are visible in the Bi NPs film Raman spectrum together with a broad shoulder attributable to a very slight oxidation of the Bi NPs (Figure 5a). The absence of considerable oxidized phase for the Bi NPs is also evident in the XRD (Figure 5b) and XPS (Figure 5c) spectra. In fact, in the former the typical peaks of the rhombohedral structure of metallic bismuth are only present, while in the latter the $\mathrm{Bi} 4 \mathrm{f}$ and O1s core-level regions are characterized by two asymmetrical peaks due to the Bi $4 f_{7 / 2}$ and $4 f_{5 / 2}$ spin-orbit components for Bi metal and by a low intensity signal for the O1s line.

The insertion of the nanoparticles in the cell cultures and in tissues and organs of living systems (mice) was performed by using a microsyringe to inject small quantitative of the solution in the extracellular liquids of the culture or in the mice's organ. Their transport, accumulation (uptake) and decay was monitored by using the X-ray fluorescence yield induced by $5 \mathrm{keV}$ electrons from a SEM in the case of cells and by an $\mathrm{X}$-ray tube operating at $45 \mathrm{kV}$ in the case of living mice [10].

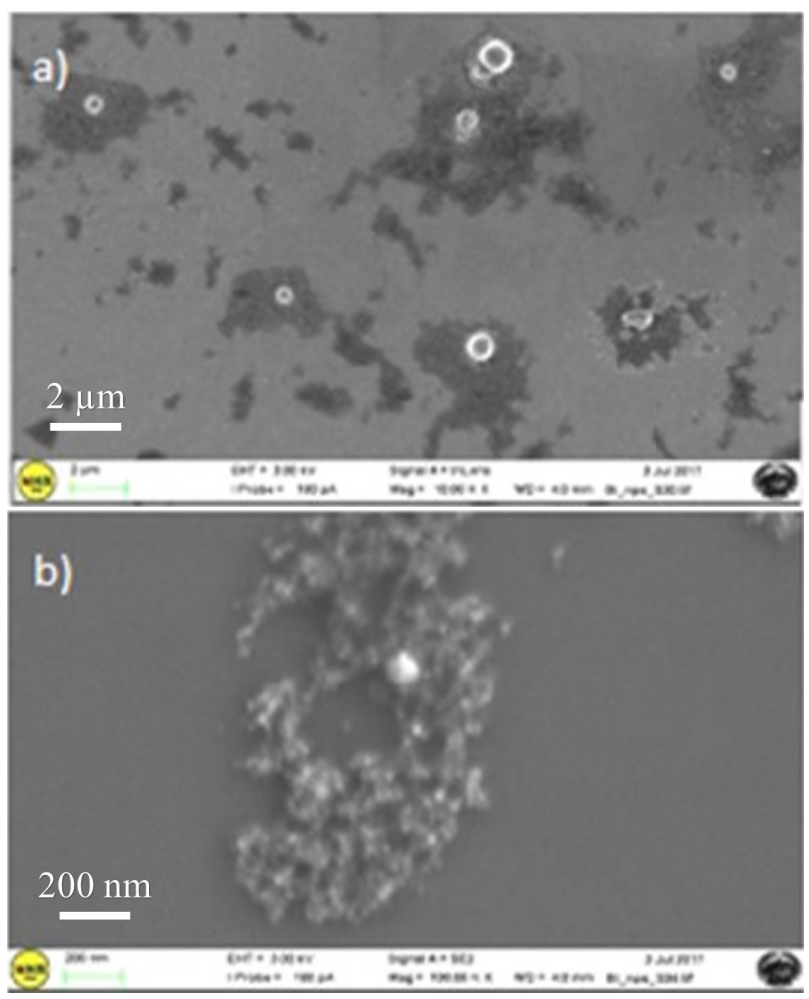

Figure 6. SEM photos of the Bi-NPs deposited (white spots) together the physiological extracellular liquid on a silicon surface at low (a) and high magnification (b).

Figure 6a shows a typical SEM investigation of Bi NPs in a physiological solution injected in the cell culture. It was observed that the Bi NPs have the possibility to go inside the cell, crossing its membrane, only if the Bi NPs dimension is lower than about 25 $\mathrm{nm}$; in fact Bi NPs with higher diameters $(\sim 50 \mathrm{~nm})$, as the one shown in Figure 6b, may be stopped on the cell membrane. Moreover the results indicate that the Bi nanoparticles are very well contrasted with respect to the surrounding low $\mathrm{Z}$ biological environment.

The use of Bi NPs as contrast medium in living tissues has been tested in mice by injecting the prepared biocompatible solution in some organs and by observing the Bi fluorescence image induced by the Bruker X-ray tube.

Figure 7 a shows the image when the solution was injected into the heart. The image displays very well the organ (observed in dark grey scale in the X-ray transmission) and the nearest blood irrigated tissues appear as very bright due to the X-ray induced fluorescence. Figure $7 \mathrm{~b}$ reports the image when the solution was directly injected in the Sx kidney. The picture illustrates very well the organ (observed in greyscale in the X-ray transmission) and the nearest blood irrigated tissues are very bright due to the $\mathrm{X}$-ray induced fluorescence. The obtained medical images are well contrasted and the interested organ shows an high spatial resolution of the zones where the Bi-NPs are localized. The aim of the study was to distinguish the interested organ in order to observe it in detail before that a successive radiotherapy treatment can be applied to the diseased tissues [11]. For comparison, Figure $7 \mathrm{c}$ reports the $\mathrm{X}$-ray image without the use of $\mathrm{Bi}-\mathrm{NPs}$.

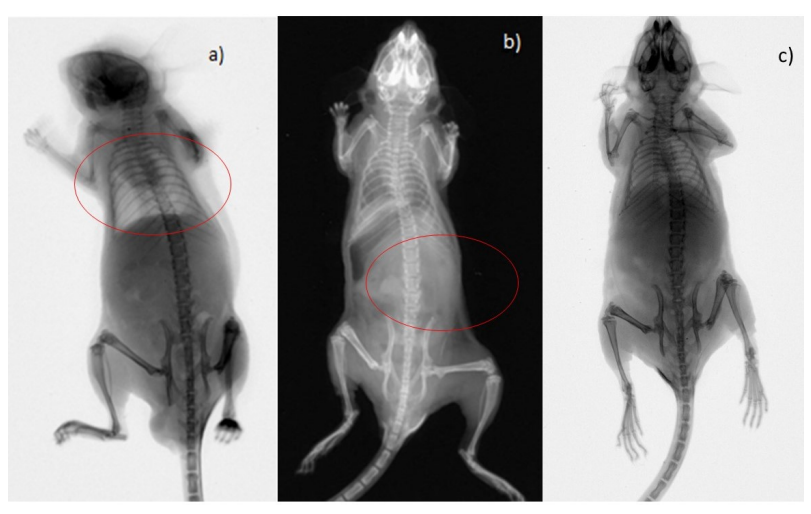

Figure 7. X-ray fluorescence image showing the AuNPs for an injection of solution in mice heart (a) and Sx kidney (b). For comparison the image without use of $\mathrm{Bi} \mathrm{NPs}(\mathrm{c})$.

\section{Discussion and Conclusions}

Our results have showed that $\mathrm{Ag}, \mathrm{Au}$ and $\mathrm{Bi}$ NPs have good biocompatibility and safety and do not affect the normal functioning of the organs. Cells (in culture) are alive after injection of the solution into their extracellular liquid and mice are also alive after the NPs injection in the blood through the tail vein or in some organs.

Measurements have been performed on the uptake and the time decay of the NPs vs. time, demonstrating that a fast uptake $(\sim 1 \div 2 \mathrm{hr})$ and a slowly decay $(\sim 12 \div 24 \mathrm{hr}$ ) occurs, depending on the nature of the used nanoparticles and on the particular organ of the 
mice. The contrasts of the fluorescence images are good and improve with the NP concentration injected in the living system.

Thanks to this type of approach it will be possible, also guided by the literature [12] to design, to functionalize and to direct the nanoparticles in the affected tumour organs and, after the diagnostics concerning their spatial localization, to proceed to their irradiation with ionizing radiations for treatments based on the traditional radiotherapy (X-rays and electrons) and on the innovative one (protons and carbon ions). In this direction we are investigating more, overall by preparing, by laser ablation, different kinds of biocompatible nanoparticles, with diverse size and shape, and by taking into consideration the application of X-ray radiotherapy whose ionization cross section increases with the atomic number such as $\mathrm{Z}^{4 \div 5}$. Of course, because of the interdisciplinary research aims, experts of physics, biology, chemistry and pharmacology are involved in this research.

\section{Acknowledgements}

This research was supported by University of Messina Research \& Mobility 2016 Project (project code RES_AND_MOB_2016_TORRISI) no. 74893496 .

Many thanks to Dr. G. Costa of MIFT Department for the support given to the preparation of this paper.

\section{References}

[1] L. Torrisi. Radiotherapy improvements by using au nanoparticles. Recent patents on Nanotechnology, 9(2):114-125, 2015. doi:10.2174/187221050902150819153355.

[2] L. Torrisi, N. Restuccia, S. Cuzzocrea, I. Paterniti, D. Ielo, S. Pergolizzi, M. Cutroneo, and L. Kovacik. Laser-produced au nanoparticles as X-ray contrast agents for diagnostic imaging. Gold Bulletin, 50(1):51-60, 2017. doi:10.1007/s13404-017-0195-y.

[3] L. Torrisi. Evaluation of the radiotherapy and proton therapy improvements using gold nanoparticles. Gold Bulletin, 50(4):299-311, 2017. doi: $10.1007 / \mathrm{s} 13404-017-0216-\mathrm{x}$.

[4] L. Torrisi. Gold nanoparticles enhancing protontherapy efficiency. Recent patents on Nanotechnology, 9(1):51-60, 2015. doi:10.2174/1872210509999141222224121.

[5] L. Torrisi and A. Torrisi. Laser ablation parameters influencing gold nanoparticle synthesis in water. Radiation Effects and Defects in Solids, 173(9-10):729739, 2018. doi:10.1080/10420150.2018.1528598.

[6] J. Fang, K. L. Stokes, J. A. Wiemann, W. L. Zhou, J. Dai, F. Chen, and C. J. O'Connor.

Microemulsion-processed bismuth nanoparticles. Materials Science and Engineering: B, 83(1-3):254-257, 2001. doi:10.1016/S0921-5107(01)00528-1.

[7] M. Maciulevičius, A. Vinčiūnas, M. Brikas, A. Butsen, N. Tarasenka, N. Tarasenko, and G. Račiukaitis. On-line characterization of gold nanoparticles generated by laser ablation in liquids. Physics Procedia, 41:531-538, 2013. doi:10.1016/j.phpro.2013.03.112.
[8] L. Torrisi, L. Silipigni, N. Restuccia, S. Cuzzocrea, M. Cutroneo, F. Barreca, B. Fazio, G. Di Marco, and S. Guglielmino. Laser-generated bismuth nanoparticles for applications in imaging and radiotherapy. Journal of Physics and Chemistry of Solids, 119:62-70, 2018. doi:10.1016/j.jpcs.2018.03.034.

[9] F. Xia, X. Xu, X. Li, L. Zhang, L. Zhang, H. Qiu, W. Wang, Y. Liu, and J. Gao. Preparation of bismuth nanoparticles in aqueous solution and its catalytic performance for the reduction of 4-nitrophenol. Industrial 63 Engineering Chemistry Research, 53(26):10576-10582, 2014. doi:10.1021/ie501142a.

[10] L. Torrisi, N. Restuccia, and I. Paterniti. Gold nanoparticles by laser ablation for x-ray imaging and protontherapy improvements. Recent patents on Nanotechnology, 12(1):59-69, 2018. doi:10.2174/1872210511666170609093433.

[11] P. K. Jain, I. H. El-Sayed, and M. A. El-Sayed. Au nanoparticles target cancer. nano today, 2(1):18-29, 2007. doi:10.1016/S1748-0132(07)70016-6.

[12] K. Huang, H. Ma, J. Liu, S. Huo, A. Kumar, T. Wei, X. Zhang, S. Jin, Y. Gan, P. C. Wang, S. He, X. Zhang, and X. Liang. Size-dependent localization and penetration of ultrasmall gold nanoparticles in cancer cells, multicellular spheroids, and tumors in vivo. ACS nano, 6(5):4483-4493, 2012. doi:10.1021/nn301282m. 